\title{
Impact of 5'-amp-activated Protein Kinase on Male Gonad and Spermatozoa Functions
}

\author{
Thi Mong Diep Nguyen ${ }^{1,2 *}$ \\ ${ }^{1}$ Physiologie de la Reproduction et des Comportements, INRA, Nouzilly, France, ${ }^{2}$ Quy Nhon University, Quy Nhon, Vietnam
}

As we already know, the male reproductive system requires less energetic investment than the female one. Nevertheless, energy balance is an important feature for spermatozoa production in the testis and for spermatozoa properties after ejaculation. The 5'-AMP-activated protein kinase, AMPK, is a sensor of cell energy, that regulates many metabolic pathways and that has been recently shown to control spermatozoa quality and functions. It is indeed involved in the regulation of spermatozoa quality through its action on the proliferation of testicular somatic cells (Sertoli and Leydig), on spermatozoa motility and acrosome reaction. It also favors spermatozoa quality through the management of lipid peroxidation and antioxidant enzymes. I review here the most

\section{OPEN ACCESS}

Edited by: Simon Rousseau,

McGill University, Canada

Reviewed by:

Jérôme Boudeau,

Centre National de la Recherche

Scientifique (CNRS), France Francisco Iñesta-Vaquera,

University of Dundee, UK

*Correspondence:

Thi Mong Diep Nguyen diepdhqn@ymail.com; thi-mong-diep.nguyen@inra.fr

Specialty section:

This article was submitted to Signaling,

a section of the journal Frontiers in Cell and Developmental Biology

Received: 23 January 2017 Accepted: 07 March 2017 Published: 23 March 2017

Citation:

Nguyen TMD (2017) Impact of 5'-amp-activated Protein Kinase on

Male Gonad and Spermatozoa Functions. Front. Cell Dev. Biol. 5:25.

doi: 10.3389/fcell.2017.00025 recent data available on the roles of AMPK in vertebrate spermatozoa functions.

Keywords: AMPK, energetic metabolism, spermatozoa, male gonad, fertility

\section{INTRODUCTION}

One of the fundamental requirements of all cells is to balance ATP consumption and ATP generation. In this regard, ATP hydrolysis is the major source of cellular energy and the study of the involvement of specific molecular mechanisms that modulate its availability is required to understand it better.

In the early 1970s, two groups postulated the existence of a regulator sensitive to the concentration of cytosolic AMP exhibiting an inhibitory effect on molecules linked to the synthesis of fatty acids: Acetyl-CoA carboxylase (ACC) (Carlson and Kim, 1973) and 3-hydroxy3-methylglutaryl coenzyme A (HMG-CoA) reductase (Beg et al., 1973). In the late 1980s, the implication of a single inhibitor of both ACC and HMG-CoA reductase, still in response to an increase in AMP concentration, was demonstrated (Carling et al., 1987). This discovery has made possible to identify this inhibitory protein as "AMP-activated protein kinase" (Carling and Hardie, 1989). Subsequent research showed that AMPK is concerned with the synthesis of fatty acids, but also with many other metabolic pathways, such as protein or carbohydrate synthesis, enabling it to play not only a key role in the intracellular detection of ATP, but also to have a more global role in maintaining energy balance in cells. From a broader point of view, AMPK was found to act as an energy regulator at both the cellular and the whole-body levels (Carling, 2004).

Recent investigation has elucidated novel biological actions of AMPK that are highly relevant to male reproduction. With the advent of new pharmacological approaches to activate AMPK in a specific manner, the possibility of targeting it in order to improve semen quality is now very close. The focus of the present review is to examine the evidences concerning the actions of AMPK in germ cells, providing new insights into the regulation of AMPK and its biological actions in male reproduction. 


\section{STRUCTURE AND EXPRESSION OF AMPK}

AMPK is a heterotrimeric serine/threonine protein kinase consisting of one catalytic subunit $(\alpha)$ and two regulatory subunits $(\beta$ and $\gamma)$ that exist as multiple isoforms and splice variants, resulting in the generation of twelve possible heterotrimeric combinations. The well-conserved genes encoding these subunits are found in the genomes of essentially all eukaryotes, including protists, fungi, plants, and animals (Hardie et al., 2003). AMPK regulates metabolic energy balance at the whole-body level by responding to hormones and nutrient signals, which leads to changes in energy homeostasis (Hardie and Ashford, 2014). Once activated by falling energy status, it promotes ATP production by increasing the activity or expression of proteins involved in catabolism (for example, glucose uptake, glycolysis, fatty acid oxidation and mitochondrial biogenesis). It does so while conserving ATP by switching off biosynthetic pathways such as the synthesis of fatty acids, triglyceride, cholesterol, glucose (via gluconeogenesis) or glycogen (Hardie, 2011).

The $\alpha$ subunit contains a conventional kinase domain containing the threonine-172 (Thr172) which is the major site phosphorylated during activation of AMPK at the N-terminus and a complex with the $\beta$ and $\gamma$ subunits at the C-terminal regions (Crute et al., 1998). The $\beta$ subunit contains two conserved regions: a CBM (carbohydrate-binding module) that is believed to bind to glycogen particles at the N-terminus (Hudson et al., 2003; Polekhina et al., 2003), and a bridge between the $\alpha$ and $\gamma$ subunits at the C-terminal (Jiang and Carlson, 1997; Thornton et al., 1998; Cheung et al., 2000). The $\gamma$ subunit carries four cystathionine- $\beta$-synthase (CBS) domains repeats which form 2 Bateman domains and is the major binding site of AMP (Bateman, 1997; Adams et al., 2004; Scott et al., 2004). AMP, ADP, or ATP can bind the AMPK $\gamma$ subunit to regulate AMPK activation through allosteric structural changes in the catalytic $\alpha$ subunit, modulating phosphorylation by upstream kinases, including STK11: serine/threonine kinase 11 [LKB1] (Hawley et al., 2003; Woods et al., 2003), calmodulin-dependent kinase kinase- $\beta$ [CaMKK $\beta$ ] (Woods et al., 2005), or TGF- $\beta$ activated kinase-1 [TAK1] (Momcilovic et al., 2006; Carling et al., 2008), and regulating the dephosphorylation of Thr172 via phosphatases (phosphatase-2 [PP2A or PP2C]) (Davies et al., 1995).

In addition to the phosphorylation of Thr172 by AMPK kinases, AMPK can be activated, more or less directly, by several pharmacological agents. Metformin and other biguanides, like phenformin (Dykens et al., 2008), activate AMPK by inhibiting Complex 1 of the respiratory chain, which, in turn, decreases intracellular ATP levels (Hardie, 2006; Hawley et al., 2010). Structurally similar to adenosine, AMPK agonist 5aminoimidazole-4-carboxamide-1-b-d-ribofuranoside (AICAR) is converted by adenosine kinase to the monophosphorylated nucleotide ZMP. ZMP binds to cystathionine $\beta$-synthase (CBS) domains of AMPK $\gamma$ subunit, allowing allosteric activation and increased phosphorylation of Thr172 (Corton et al., 1995). Moreover, some natural compounds, like resveratrol, which is found, for instance, in the skin of red grapes (Baur et al.,
2006; Zang et al., 2006), activate AMPK and have a beneficial effect on metabolic diseases comparable to those of AICAR and metformin. This compound acts in a quick fashion by inhibiting the F1F0 mitochondrial ATPase (Hawley et al., 2010) and seems to activate Sirtuins (SIRT1) through increased $\mathrm{NAD}^{+}$levels, induced themselves by AMPK activity (Canto and Auwerx, 2010; Um et al., 2010). Unlike AICAR and metformin, A-769662, a new activator of AMPK (Scott et al., 2014), appears to use a different molecular mechanism to activate AMPK. As it does not increase cellular ADP/ATP or AMP/ATP ratios, A-769662 is considered to directly activate AMPK in cells, expressing an AMP-insensitive mutant (Hawley et al., 2010). Moreover, it does not displace AMP from its binding sites on the $\gamma$ subunit, suggesting that it binds at a site different from AMP even though, like AMP, it causes both allosteric activation and protection against Thr172 dephosphorylation (Cool et al., 2006; Goransson et al., 2007; Sanders et al., 2007; Scott et al., 2008). A-769662 is also selective for activation of $\beta 1$ rather than $\beta 2$ complexes, and its effects are abolished by an S108 mutation in $\beta 1$ that prevents the autophosphorylation of that serine residue, suggesting that the binding site involved the $\beta$ subunit (Sanders et al., 2007; Scott et al., 2008, 2014). The activity of AMPK is inhibited by (6-[4-(2-piperidin-1-yl-ethoxy)-phenyl)]3-pyridin4-yl-pyrazolo[1,5-a] pyrimidine (Zhou et al., 2001) known as compound $\mathrm{C}$ or dorsomorphin. It can also block the uptake of AICAR into cells and thus inhibit AICAR stimulatory effects (Fryer et al., 2002). In addition, when incubated with either AICAR or metformin, compound $\mathrm{C}$ hinders the inactivation of acetyl CoA carboxylase (ACC) (King et al., 2006).

Among the signaling proteins having a strong impact on the regulation of the functions of somatic and germ cells, AMPK is a crucial cellular energy sensor. Relationships between metabolism and fertility are well established, particularly in the female. Recent discoveries show that AMPK is also present in male gonad and spermatozoa in different species [Caenorhabditis elegans (Lee et al., 2008), oyster (Guevelou et al., 2013), chicken (Nguyen et al., 2014), boar (Hurtado de Llera et al., 2012, 2013), mouse (Tanwar et al., 2012; Tartarin et al., 2012), stallion (Cordova et al., 2014), and human Calle-Guisado et al., 2016; Shabani Nashtaei et al., 2016]. It is these roles in male gonad and spermatozoa that I will now describe.

\section{ROLE OF AMPK IN MALE GONAD AND SPERM PRODUCTION}

The male reproductive system in mammals consists of the testis and a series of ducts and glands. These organs produce semen, which contains spermatozoa as well as other components. Spermatozoa differentiate from germ stem cells in the testis and are transported along the reproductive ducts: the epididymes, the vas deferens, the ejaculatory duct and the urethra (Caroppo, 2011). Testis not only produce gametes (spermatogenesis), but also steroid hormones (steroidogenesis) (Rato et al., 2012; Alves et al., 2013) that control the physiological and behavioral characteristics of male reproduction. Sertoli cells play pivotal roles in $1 /$ the production and differentiation of germ cells 
as well as in $2 /$ estrogen and specific proteins synthesis (ABP (androgen-binding protein), SCF (stem cell factor), AMH (antiMullerian hormone), etc.).

AMPK has been detected for the first time in male germinal cells in 2000, and $\alpha 1$ and $\gamma 1$ isoforms at a higher level than $\alpha 2$ or $\gamma 3$ isoforms in rat testis (Cheung et al., 2000). AMPK activity in male germinal cells was then presented in 2008 by Towler et al. The kinase upstream of AMPK, called LKB1s, plays a crucial role in male fertility in mice (Towler et al., 2008). Indeed, the absence of LKB1 [LKB1 knockout (KO)] and $\mathrm{AMPK} \alpha 1$ [AMPK $\alpha 1 \mathrm{KO}$ ] genes in mice decreases spermatozoa production, leading to a reduction of mature spermatozoa in the epididymis in parallel with an increase of the amount of morphologically abnormal, non-motile spermatozoa (Towler et al., 2008), as well as a reduction in mitochondrial membrane potential, a lower basal oxygen consumption, and a decrease in spermatozoa motility (Tartarin et al., 2012). The AMPK $\alpha 1$ subunit is also localized in mouse Sertoli cells (Bertoldo et al., 2016) where the AMPK/mTOR signaling pathway is involved in the regulation of autophagy and apoptosis induced by toxic substances in Sertoli cells (Duan et al., 2016). AMPK activation leads to an increase of lactate production, in response to an increase in glucose uptake and accordingly to increased levels of GLUT1 (glucose transporter 1) and MCT4 (monocarboxylate transporter 4) (Galardo et al., 2007; Um et al., 2010). Specific deletion of the AMPK 1 gene in the Sertoli cells in mice (ScAMPK $\alpha 1 \mathrm{KO}$ mice) has led to a $25 \%$ reduction in male fertility, associated with abnormal spermatozoa with a thin head and dysregulated energy metabolism with altered lactate, lipid, and ATP production (Bertoldo et al., 2016). In rat Leydig cells, the use of the AMPK activator resveratrol decreased testosterone secretion through a reduction in cholesterol transport into the mitochondria and decreased conversion of progesterone into androstenedione (Svechnikov et al., 2009). In chicken testis, the AMPK $\alpha 1 / 2$ subunit is found in seminiferous tubules and in Leydig cells, AMPK $\beta 1 / 2$ and AMPK $\gamma 1$ subunits in seminiferous tubules as well, and AMPK $\gamma 3$ in Leydig cells (Faure et al., 2016). In chicken (7-10 weeks old), metformin induced a decrease in testis weight, in the proliferative activity of Sertoli cells and in germ cells population, but not in spermatogonia population. Metformin also decreased testosterone levels in chicken Sertoli cells (Faure et al., 2016). These data suggest that the AMPK pathway is involved in the nutritional modulation of the male gonad and spermatozoa production.

Furthermore, the presence of AMPK, especially in its active form, phospho-AMPK-Thr172, was highlighted in spermatozoa of boar, stallion, mouse, chicken and human (Hurtado de Llera et al., 2012, 2013; Tartarin et al., 2012; Cordova et al., 2014; Nguyen et al., 2014; Calle-Guisado et al., 2016; Shabani Nashtaei et al., 2016). In boar, the phospho-AMPK form is mainly localized in the acrosome, the sub-equatorial segment of the head and in the intermediate part (Hurtado de Llera et al., 2013). In stallion, phospho-AMPK has been located in the main piece of the flagellum and the sub-equatorial region of the head (Cordova et al., 2014). In human, AMPK protein is localized all along the spermatozoa, in the entire acrosome, the midpiece, and along the tail of the flagellum (Calle-Guisado et al., 2016;
Shabani Nashtaei et al., 2016). In contrast, phospho-AMPK is localized only at the most apical part of the acrosome region and at the spermatozoa tail, and lightly in the post-acrosomal region of the head and in the midpiece region of the tail (CalleGuisado et al., 2016). In chicken, AMPK is mainly localized in the acrosome, the intermediate part, and the whole flagellum, but phospho-AMPK is especially present in the flagellum and the acrosome, and with a much lower intensity in the intermediate part (Nguyen et al., 2014). The localization of phospho-AMPK in spermatozoa flagellum of several species suggests that it acts through the phosphorylation of protein substrates involved in the functioning of the axoneme central apparatus that is essential for flagellar motility. The mechanism could be similar to that demonstrated for a testicular AMPK-related kinase: TSSK2 (Testis-Specific Serine Kinase 2), a Ser/Thr protein kinase close to AMPK (Scott et al., 2008). In vitro, TSKK2 phosphorylates SPAG16L, a protein of the axoneme central apparatus which is essential for mouse spermatozoa flagellar motility (Moreno et al., 2008). By contrast, in chicken, phospho-AMPK-Thr-172 is mainly present in the flagellum and the acrosome, and much less in the intermediate part (Nguyen et al., 2014). These results are in agreement with the fact that the ATP production is not limited to the spermatozoa intermediate piece (mitochondrial respiration) but may also occur at other places via anaerobic glycolysis (Zhou et al., 2001; Scott et al., 2014). Therefore, the AMPK distribution profile in spermatozoa cells suggests that, depending on the species, it promotes in different ways both motility and acrosome reaction. The links between differences in subcellular localization of AMPK and spermatozoa biological characteristics of different species, however, have not yet been defined. In invertebrates, AMPK has also been highlighted in 2013 in oyster's male and female germ cells, where it is involved in the glucose and fatty acids metabolism (Guevelou et al., 2013).

\section{ROLE OF AMPK IN SPERMATOZOA FUNCTIONS}

The key role of AMPK in the control of cell energy homeostasis has placed it in a position of an important kinase regarding the regulation of those spermatozoa functions. Indeed, these functions, such as motility, acrosome reaction, and fertilization, are very dependent on energy levels (Hurtado de Llera et al., 2012, 2013; Tartarin et al., 2012; Cordova et al., 2014; Nguyen et al., 2014, 2015; Calle-Guisado et al., 2016; Shabani Nashtaei et al., 2016). AMPK activation in spermatozoa by pharmacological factors favors motility (Nguyen et al., 2014, 2015; Hurtado de Llera et al., 2015; Shabani Nashtaei et al., 2016), acrosome reaction and spermatozoa ability to successfully fertilize the oocyte (Nguyen et al., 2014, 2015). AMPK is also implicated in the mitochondrial membrane potential, spermatozoa plasma membrane fluidity and organization, and acrosome integrity (Hurtado de Llera et al., 2013; Shabani Nashtaei et al., 2016). By contrast, pharmacological inhibition of AMPK, for example with compound $\mathrm{C}$, leads to a reduction in motility parameters such as the percentages of motile and rapid spermatozoa or their curvilinear velocity and average path velocity, in boar, 
chicken, and human spermatozoa (Hurtado de Llera et al., 2012; Nguyen et al., 2014, 2015; Calle-Guisado et al., 2016; Shabani Nashtaei et al., 2016). Unexpectedly, compound C does not affect spermatozoa functions in stallion (Cordova et al., 2014).

Different AMPK activators exhibit various effects on spermatozoa functions. Metformin promotes no deleterious effect in mouse spermatozoa quality, viability, or integrity of the acrosome membrane, except for a slight reduction in spermatozoa motility when used at high concentration $(5 \mathrm{mM})$, due to a reduction of active mitochondria (Bertoldo et al., 2014). When mouse semen is cryopreserved, spermatozoa viability and membrane integrity is increased by pre-incubating it with metformin before freezing. This also significantly increases the percentage of fertilized oocytes (Bertoldo et al., 2014). Using fresh or frozen-thawed chicken spermatozoa demonstrated that exposure to metformin significantly improves AMPK phosphorylation, spermatozoa motility, viability, and percentage of spermatozoa able to undergo a successful acrosome reaction (Nguyen et al., 2015). The use of metformin in cryopreservation protocols actually seems to have an overall beneficial effect on the quality of cryopreserved semen, improving its quality and fertilization ability, in response to the activation of the AMPK pathway. The other AMPK activators, AICAR and A-769662, exhibit the same beneficial effects as metformin. AICAR increases chicken spermatozoa quality (motility, viability and acrosome reaction) (Nguyen et al., 2014, 2015), and A-769662 improves boar spermatozoa motility (Hurtado de Llera et al., 2015). Supporting a relevant role of AMPK in spermatozoa functions, Tartarin et al. have shown a significant reduction in the fertilizing capacity in mouse epididymal spermatozoa lacking the AMPK catalytic subunit $\alpha 1$ gene [AMPK $\alpha 1 \mathrm{KO}$ ]. This is due to asthenozoospermia and higher rate of morphological and functional abnormalities linked to mitochondrial dysgenesis. There is, however, no changes in testis morphology or spermatozoa production rate. Furthermore, the increase in intra-testicular cholesterol and testosterone, in parallel with a decrease in LH and FSH levels in KO mice, show that the lack of AMPK activation seems to result in an increase of androgens levels, which can negatively influence spermatozoa quality, and consequently, male fertility (Tartarin et al., 2012). Regarding human germ cells, it has been recently shown that resveratrol could activate and phosphorylate AMPK in frozen-thawed spermatozoa, and thus help in restoring their functions, and that compound C had opposite effects (Shabani Nashtaei et al., 2016).

\section{ROLE OF AMPK IN LIPID PEROXIDATION AND ANTIOXIDANT ENZYMES}

Oxygen-free radicals, also known as reactive oxygen species (ROS), can be either harmful or beneficial. They are needed for the maturation of cellular structures and help the defense system, supporting cell proliferation and survival pathways (Sundaresan et al., 1995; Echtay et al., 2002; Pacher et al., 2007). But if they are produced in excess, they can damage the cells components and hamper physiological functions (Droge, 2002; Fulda et al., 2010; Kong and Lin, 2010; Martin, 2010; Navarro and Boveris, 2010).

In male reproduction, it has been observed that ROS can disrupt the spermatozoa response to calcium signaling and inhibit the ionophore-induced sperm-oocyte fusion (Aitken and Clarkson, 1987). This could be the result of decreased plasma membrane fluidity and of membrane-bound enzymes and/or ion channels malfunctions, as a consequence of peroxidation (Aitken et al., 1989). ROS have a negative effect on motility which might be due to the depletion of ATP by $\mathrm{H}_{2} \mathrm{O}_{2}$ inhibiting oxidative phosphorylation and/or glycolysis, and thus hindering ATP restoration (deLamirande and Gagnon, 1992a). This process could happen by decreasing phosphorylation of axonemal proteins involved in spermatozoa motility (de Lamirande and deLamirande and Gagnon, 1992b), by depressing glycolytic flux (Baumber et al., 2000), or through products of lipid peroxidation like malondialdehyde or 4-hydroxynonenol (4HN), inhibiting anaerobic glycolysis, DNA, RNA, and protein synthesis (Comporti, 1989).

AMPK appears to be involved in the mechanisms protecting the cells against toxic effects of ROS. Although there are still unknown factors or difficulties in assessing significance of ROS, the goal of many researches is to prevent or counteract their deleterious effects. In forms of diabetes in which the activity of AMPK is strongly impaired, reactivation of AMPK could counteract the negative effects of oxidative stress linked to this metabolic disease (Ceolotto et al., 2007). Resveratrol, by activating AMPK, reduces superoxide anion and protein carbonyl in type II diabetic mice (Chang et al., 2011), and metformin reduces NAFLD in obese $\mathrm{Ob} / \mathrm{Ob}$ mice submitted to fat diet (Viollet et al., 2012). It is also possible that the AMPK activation caused by resveratrol helps the mitochondria in its defense against oxidative stress by inhibiting ROS generation and permeability transition; it could do that while playing a part in the downstream inhibitory phosphorylation of GSK3b at the same time (Shin et al., 2009). The positive effect of metformin on steatosis depends on the activation of AMPK secondary to the inhibition of complex I of the respiratory chain and the generated energy deficiency (augmented AMP/ATP ratio). An "antioxidant" effect of AMPK has been shown in different systems, and in particular in tumor cells deficient in LKB1 of lung adenocarcinoma. In this model, AMPK may increase the levels of intracellular NADPH in different ways: by the activation of the oxidation of fatty acids and by the inhibition of fatty acid synthesis to neutralize the cytotoxic ROS (Jeon et al., 2012). The antioxidant defense and glutathione regeneration efficiency is greatly improved by NADPH since it is a very important source of reducing equivalent (Fico et al., 2004). Antioxidant enzymes depending on NADPH, like those of the thioredoxin and glutaredoxin classes, are heavily involved in the maintenance of redox homeostasis due to the regulation of thiol-disulfide exchange (Kalinina et al., 2008; Myers and Myers, 2009). While under oxidative stress conditions, AMPK is activated and helps to increase NADPH production through pentose phosphate pathway by starting the glytolytic flux (Wu and Wei, 2012).

In the male reproductive function, a link between AMPK and antioxidant mechanisms also exists. Thus, in the testis of 
diabetic and obese rats, the use of metformin induces a reduction in the levels of lipid peroxidation (LPO) accompanied by a stimulation of steroidogenesis, a restoration of spermatogenesis, and an increase in spermatozoa concentration and motility (Fang et al., 2012; Nasrolahi et al., 2013). In recent years, the involvement of AMPK in the regulation of ROS or LPO concentrations and the activity of the main antioxidant enzymes in spermatozoa has been demonstrated (Nguyen et al., 2015). The addition of an AMPK activator, metformin or AICAR, before cryopreservation increases the phosphorylation of AMPK while reducing the production of ROS and LPO in chicken frozen/thawed spermatozoa. At the same time, AICAR and metformin increase the activity of antioxidant enzymes superoxide dismutase (SOD), glutathione peroxidase (GPx) and glutathione reductase (GR) in the cryopreserved semen. It has been observed that human spermatozoa incubated with AMPK activator resveratrol have decreased ROS levels $\left(\mathrm{O}_{2}^{-}\right.$ and $\mathrm{H}_{2} \mathrm{O}_{2}$ ), while AMPK inhibitor increased them (Shabani Nashtaei et al., 2016). Resveratrol had the same effects on mouse spermatozoa, where a $15 \mu \mathrm{g} / \mathrm{mL}$ dosage significantly decreased ROS (Mojica-Villegas et al., 2014). This shows that
AMPK activity is limiting ROS production in endothelial cells (Kim et al., 2008), in mice endothelial progenitor cells (by restoring manganese superoxide dismutase (MnSOD) levels) (Wang et al., 2011), and in cryopreserved chicken spermatozoa (Nguyen et al., 2015). However, the mechanisms by which AMPK activators would act on ROS and antioxidant enzyme systems in spermatozoa are still unclear.

AMPK is known to be involved in the renewal of cellular mitochondrial content by stimulating the degradation of defective mitochondria through ULK1/2 (Unc51-like kinase) on the one hand, and by activating biogenesis new mitochondria via PGC1 $\alpha$ on the other hand, and thus, indirectly, on antioxidant systems (Canto et al., 2009; Aquilano et al., 2010). But since AMPK stimulation of the regeneration of NADPH (Williams and Ford, 2004) by the pentose phosphate pathway has been demonstrated in mammalian spermatozoa, it probably contributes to the antioxidant system through stimulation of GPx, and could also, as a result, have a role in this function. AMPK could have a part in the up-regulation of several antioxidant enzymes (Colombo and Moncada, 2009; Calegari et al., 2011). It can directly phosphorylate the

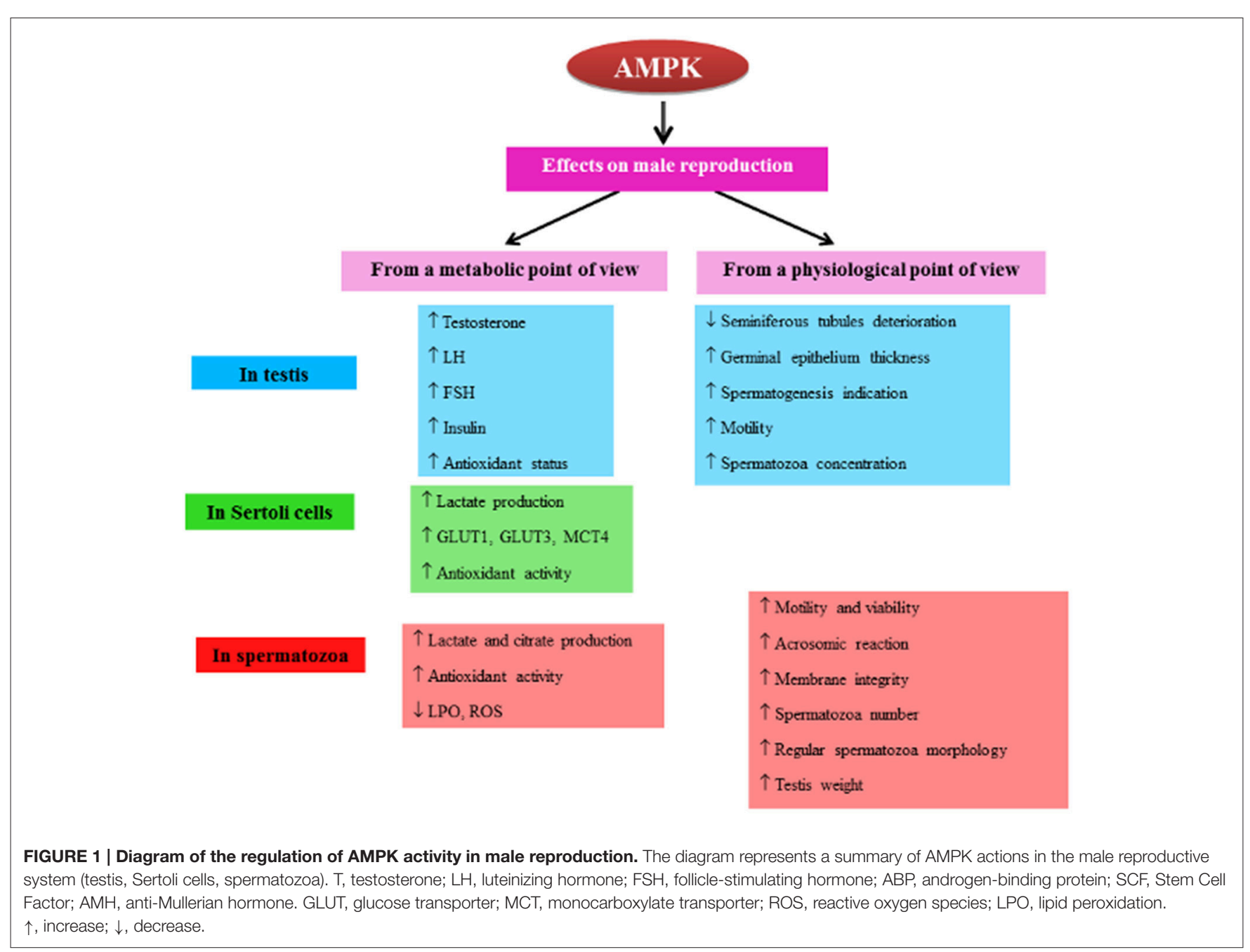


forkhead transcription factor (FOXO) to promote its nuclear translocation and the formation of subsequent transcription activation complex (Greer et al., 2007). Activating AMPK-FOXO pathway helps up-regulating the expression of thioredoxin and peroxiredoxin and thus reduces oxidant-induced ROS (Chiribau et al., 2008; Li et al., 2009). Through usage of endothelial cells with silenced AMPK $\alpha 1$ subunit, it has also been shown that AMPK induced the expression of genes playing a role in antioxidant defense (like MnSOD, catalase, $\gamma$-glutamylcysteine synthase, or thioredoxin) (Colombo and Moncada, 2009). This procedure goes along with a decreased mitochondrial content and increased ROS, suggesting that AMPK $\alpha 1$ involvement in endothelial cells happens in both mitochondrial content and antioxidant defenses. This method also significantly reduced FOXO3a at the protein and mRNA levels, providing further evidence of its role in AMPK antioxidant defense regulation.

I believe that these data will be useful to develop and improve the methodologies of semen handling and storage. Moreover, the positive effects of AMPK stimulation on the functions of spermatozoa remain after cryopreservation (chicken, mice), which supports the hypothesis of its "protector" role on gametes after exposure to extreme heat and osmotic stress conditions encountered during the freeze/thaw process (Bertoldo et al., 2014; Nguyen et al., 2015).

\section{SPERMATOZOA AMPK: A LINK BETWEEN ENERGY METABOLISM AND FERTILITY}

The spermatozoa energy in the form of ATP is mainly obtained from aerobic metabolic (involving the citric acid cycle and oxidative phosphorylation) and/or anaerobic pathways through glycolysis. The metabolic pathways of spermatozoa are mainly localized in mitochondria (Krebs cycle, oxidative phosphorylation) which are found themselves in the intermediate part, the cytoplasm, the fibrous sheath of the flagellum, and the acrosome (Ferramosca and Zara, 2014). AMPK helps to regulate the mitochondrial membrane potential in spermatozoa of boars (Hurtado de Llera et al., 2013) and mice (Tartarin et al., 2012; Bertoldo et al., 2014). An increase in the production of ATP and lactate in spermatozoa cells was observed after the stimulation of AMPK by pharmacological activators (metformin

\section{REFERENCES}

Adams, J., Chen, Z. P., Van Denderen, B. J., Morton, C. J., Parker, M. W., Witters, L. A., et al. (2004). Intrasteric control of AMPK via the gammal subunit AMP allosteric regulatory site. Protein Sci. 13, 155-165. doi: 10. 1110/ps.03340004

Aitken, R. J., and Clarkson, J. S. (1987). Cellular basis of defective sperm function and its association with the genesis of reactive oxygen species by human spermatozoa. J. Reprod. Fertil. 81, 459-469. doi: 10.1530/jrf.0.0810459

Aitken, R. J., Clarkson, J. S., and Fishel, S. (1989). Generation of reactive oxygen species, lipid peroxidation, and human sperm function. Biol. Reprod. 41, 183-197. doi: 10.1095/biolreprod41.1.183

Alves, M. G., Rato, L., Carvalho, R. A., Moreira, P. I., Socorro, S., and Oliveira, P. F. (2013). Hormonal control of Sertoli cell metabolism or AICAR) in chicken (Nguyen et al., 2014, 2015). AICAR also stimulates the production of citrate, an intermediate in the Krebs cycle. In the particular case of cryopreservation, after thawing, many spermatozoa die or are unable to move due to alterations of the flagellum or the intermediate piece, or unable to accomplish the acrosome reaction. All these alterations result in a decreased fertility. In these conditions, spermatozoa require a very large amount of energy to restore their fertilizing capacity. The addition of metformin or AICAR before cryopreservation would restore a portion of the energy needed by stimulating the anaerobic metabolism and, for AICAR, the aerobic metabolism (Nguyen et al., 2015). AMPK is therefore essential to keep spermatozoa at the energy level necessary for the conduct of fertilization, especially in "stressful" conditions.

\section{CONCLUSIONS}

All the previously published data and my own results suggest an important role of AMPK in the development of the male gonad and spermatozoa production (Figure 1). It is also involved in spermatozoa functions: in motility and acrosome reaction as well as in the energy and redox capacities in order to regulate cellular processes necessary for successful fertilization. Its role in the regulation of antioxidant defenses of the cell particularly affects the in vitro retention capacity, which is especially solicited during the freezing of semen. From a broader point of view, AMPK could become a therapeutic target for limiting infertility and improving biotechnologies of artificial insemination and semen conservation. Further work is needed to study the involvement of AMPK in human male reproductive function.

\section{AUTHOR CONTRIBUTIONS}

$\mathrm{TN}$ collected the material and wrote the manuscript.

\section{ACKNOWLEDGMENTS}

The author thanks Dr Yves Combarnous from INRACNRS laboratory "Physiologie de la Reproduction et des Comportements", F-37380 Nouzilly, France, for his help in critically reading the manuscript. regulates spermatogenesis. Cell. Mol. Life Sci. 70, 777-793. doi: 10.1007/s00018-012-1079-1

Aquilano, K., Vigilanza, P., Baldelli, S., Pagliei, B., Rotilio, G., and Ciriolo, M. R. (2010). Peroxisome proliferator-activated receptor $\gamma$ co-activator $1 \alpha \quad($ PGC-1 $\alpha)$ and sirtuin 1 (SIRT1) reside in mitochondria: possible direct function in mitochondrial biogenesis. J. Biol. Chem. 285, 21590-21599. doi: 10.1074/jbc.M109.070169

Bateman, A. (1997). The structure of a domain common to archaebacteria and the homocystinuria disease protein. Trends Biochem. Sci. 22, 12-13. doi: 10.1016/S0968-0004(96)30046-7

Baumber, J., Ball, B. A., Gravance, C. G., Medina, V., and Davies-Morel, M. C. (2000). The effect of reactive oxygen species on equine sperm motility, viability, acrosomal integrity, mitochondrial membrane potential, and membrane lipid peroxidation. J. Androl. 21, 895-902. doi: 10.1002/j.1939-4640.2000.tb03420.x 
Baur, J. A., Pearson, K. J., Price, N. L., Jamieson, H. A., Lerin, C., Kalra, A., et al. (2006). Resveratrol improves health and survival of mice on a high-calorie diet. Nature 444, 337-342. doi: 10.1038/nature05354

Beg, Z. H., Allmann, D. W., and Gibson, D. M. (1973). Modulation of 3-hydroxy3-methylglutaryl coenzyme A reductase activity with cAMP and wth protein fractions of rat liver cytosol. Biochem. Biophys. Res. Commun. 54, 1362-1369. doi: 10.1016/0006-291X(73)91137-6

Bertoldo, M. J., Guibert, E., Faure, M., Guillou, F., Rame, C., Nadal-Desbarats, L., et al. (2016). Specific deletion of AMP-activated protein kinase ( $\alpha 1 \mathrm{AMPK})$ in mouse Sertoli cells modifies germ cell quality. Mol. Cell. Endocrinol. 423, 96-112. doi: 10.1016/j.mce.2016.01.001

Bertoldo, M. J., Guibert, E., Tartarin, P., Guillory, V., and Froment, P. (2014). Effect of metformin on the fertilizing ability of mouse spermatozoa. Cryobiology 68, 262-268. doi: 10.1016/j.cryobiol.2014.02.006

Calegari, V. C., Zoppi, C. C., Rezende, L. F., Silveira, L. R., Carneiro, E. M., and Boschero, A. C. (2011). Endurance training activates AMP-activated protein kinase, increases expression of uncoupling protein 2 and reduces insulin secretion from rat pancreatic islets. J. Endocrinol. 208, 257-264. doi: 10.1530/JOE-10-0450

Calle-Guisado, V., de Llera, A. H., Martin-Hidalgo, D., Mijares, J., Gil, M. C., Alvarez, I. S., et al. (2016). AMP-activated kinase in human spermatozoa: identification, intracellular localization, and key function in the regulation of sperm motility. Asian J. Androl. doi: 10.4103/1008-682x.185848. [Epub ahead of print]

Canto, C., and Auwerx, J. (2010). AMP-activated protein kinase and its downstream transcriptional pathways. Cell. Mol. Life Sci. 67, 3407-3423. doi: 10.1007/s00018-010-0454-Z

Canto, C., Gerhart-Hines, Z., Feige, J. N., Lagouge, M., Noriega, L., Milne, J. C., et al. (2009). AMPK regulates energy expenditure by modulating $\mathrm{NAD}^{+}$metabolism and SIRT1 activity. Nature 458, 1056-1060. doi: 10.1038 /nature 07813

Carling, D. (2004). The AMP-activated protein kinase cascade-a unifying system for energy control. Trends Biochem. Sci. 29, 18-24. doi: 10.1016/j.tibs.2003.11.005

Carling, D., and Hardie, D. G. (1989). The substrate and sequence specificity of the AMP-activated protein kinase. Phosphorylation of glycogen synthase and phosphorylase kinase. Biochim. Biophys. Acta 1012, 81-86. doi: 10.1016/0167-4889(89)90014-1

Carling, D., Sanders, M. J., and Woods, A. (2008). The regulation of AMPactivated protein kinase by upstream kinases. Int. J. Obes. 32(Suppl. 4), S55S59. doi: 10.1038/ijo.2008.124

Carling, D., Zammit, V. A., and Hardie, D. G. (1987). A common bicyclic protein kinase cascade inactivates the regulatory enzymes of fatty acid and cholesterol biosynthesis. FEBS Lett. 223, 217-222. doi: 10.1016/0014-5793(87)80292-2

Carlson, C. A., and Kim, K. H. (1973). Regulation of hepatic acetyl coenzyme a carboxylase by phosphorylation and dephosphorylation. J. Biol. Chem. 248, $378-380$

Caroppo, E. (2011). "Male reproductive medicine: anatomy and physiology," in An Introduction to Male Reproductive Medicine, ed C. Niederberger (Cambrige, MA; New York, NY: Cambridge University Press), 1-28.

Ceolotto, G., Gallo, A., Papparella, I., Franco, L., Murphy, E., Iori, E., et al. (2007). Rosiglitazone reduces glucose-induced oxidative stress mediated by $\mathrm{NAD}(\mathrm{P}) \mathrm{H}$ oxidase via AMPK-dependent mechanism. Arterioscler. Thromb. Vasc. Biol. 27, 2627-2633. doi: 10.1161/atvbaha.107.155762

Chang, C. C., Chang, C. Y., Wu, Y. T., Huang, J. P., Yen, T. H., and Hung, L. M. (2011). Resveratrol retards progression of diabetic nephropathy through modulations of oxidative stress, proinflammatory cytokines, and AMPactivated protein kinase. J. Biomed. Sci. 18:47. doi: 10.1186/1423-0127-18-47

Cheung, P. C., Salt, I. P., Davies, S. P., Hardie, D. G., and Carling, D. (2000). Characterization of AMP-activated protein kinase gamma-subunit isoforms and their role in AMP binding. Biochem. J. 346(Pt 3), 659-669. doi: 10.1042/bj3460659

Chiribau, C. B., Cheng, L., Cucoranu, I. C., Yu, Y. S., Clempus, R. E., and Sorescu, D. (2008). FOXO3A regulates peroxiredoxin III expression in human cardiac fibroblasts. J. Biol. Chem. 283, 8211-8217. doi: 10.1074/jbc.M710610200

Colombo, S. L., and Moncada, S. (2009). AMPKalpha1 regulates the antioxidant status of vascular endothelial cells. Biochem. J. 421, 163-169. doi: 10.1042/BJ20090613
Comporti, M. (1989). Three models of free radical-induced cell injury. Chem. Biol. Interact. 72, 1-56. doi: 10.1016/0009-2797(89)90016-1

Cool, B., Zinker, B., Chiou, W., Kifle, L., Cao, N., Perham, M., et al. (2006). Identification and characterization of a small molecule AMPK activator that treats key components of type 2 diabetes and the metabolic syndrome. Cell Metab. 3, 403-416. doi: 10.1016/j.cmet.2006.05.005

Cordova, A., Strobel, P., Vallejo, A., Valenzuela, P., Ulloa, O., Burgos, R. A., et al. (2014). Use of hypometabolic TRIS extenders and high cooling rate refrigeration for cryopreservation of stallion sperm: presence and sensitivity of 5' AMP-activated protein kinase (AMPK). Cryobiology 69, 473-481. doi: 10.1016/j.cryobiol.2014.10.008

Corton, J. M., Gillespie, J. G., Hawley, S. A., and Hardie, D. G. (1995). 5-aminoimidazole-4-carboxamide ribonucleoside. A specific method for activating AMP-activated protein kinase in intact cells? Eur. J. Biochem. 229, 558-565. doi: 10.1111/j.1432-1033.1995.tb20498.x

Crute, B. E., Seefeld, K., Gamble, J., Kemp, B. E., and Witters, L. A. (1998). Functional domains of the $\alpha 1$ catalytic subunit of the AMP-activated protein kinase. J Biol. Chem. 273, 35347-35354. doi: 10.1074/jbc.273.52.35347

Davies, S. P., Helps, N. R., Cohen, P. T., and Hardie, D. G. (1995). 5'-AMP inhibits dephosphorylation, as well as promoting phosphorylation, of the AMPactivated protein kinase. Studies using bacterially expressed human protein phosphatase-2C $\alpha$ and native bovine protein phosphatase-2AC. FEBS Lett. 377, 421-425. doi: 10.1016/0014-5793(95)01368-7

Droge, W. (2002). Free radicals in the physiological control of cell function. Physiol. Rev. 82, 47-95. doi: 10.1152/physrev.00018.2001

Duan, P., Hu, C., Quan, C., Yu, T., Zhou, W., Yuan, M., et al. (2016). 4Nonylphenol induces apoptosis, autophagy and necrosis in Sertoli cells: involvement of ROS-mediated AMPK/AKT-mTOR and JNK pathways. Toxicology 341-343, 28-40. doi: 10.1016/j.tox.2016.01.004

Dykens, J. A., Jamieson, J., Marroquin, L., Nadanaciva, S., Billis, P. A., and Will, Y. (2008). Biguanide-induced mitochondrial dysfunction yields increased lactate production and cytotoxicity of aerobically-poised HepG2 cells and human hepatocytes in vitro. Toxicol. Appl. Pharmacol. 233, 203-210. doi: 10.1016/j.taap.2008.08.013

Echtay, K. S., Roussel, D., St-Pierre, J., Jekabsons, M. B., Cadenas, S., Stuart, J. A., et al. (2002). Superoxideactivates mitochondrial uncouplingproteins. Nature 415, 96-99. doi: 10.1038/415096a

deLamirande, E., and Gagnon, C. (1992a). Reactive oxygen species and human spermatozoa. II. Depletion of adenosine triphosphate plays an important role in the inhibition of sperm motility. J. Androl. 13, 379-386.

deLamirande, E., and Gagnon, C. (1992b). Reactive oxygen species and human spermatozoa. I. Effects on the motility of intact spermatozoa and on sperm axonemes. J. Androl. 13, 368-378.

Fang, X., Xu, Q. Y., Jia, C., and Peng, Y. F. (2012). Metformin improves epididymal sperm quality and antioxidant function of the testis in diet-induced obesity rats. Zhonghua Nan KeXue 18, 146-149.

Faure, M., Guibert, E., Alves, S., Pain, B., Rame, C., Dupont, J., et al. (2016). The insulin sensitiser metformin regulates chicken Sertoli and germ cell populations. Reproduction 151, 527-538. doi: 10.1530/REP-15-0565

Ferramosca, A., and Zara, V. (2014). Bioenergetics of mammalian sperm capacitation. Biomed. Res. Int. 2014:902953. doi: 10.1155/2014/902953

Fico, A., Paglialunga, F., Cigliano, L., Abrescia, P., Verde, P., Martini, G., et al. (2004). Glucose-6-phosphate dehydrogenase plays a crucial role in protection from redox-stress-induced apoptosis. Cell Death Differ. 11, 823-831. doi: $10.1038 /$ sj.cdd. 4401420

Fryer, L. G., Parbu-Patel, A., and Carling, D. (2002). Protein kinase inhibitors block the stimulation of the AMP-activated protein kinase by 5-amino-4-imidazolecarboxamide riboside. FEBS Lett. 531, 189-192. doi: 10.1016/S0014-5793(02)03501-9

Fulda, S., Gorman, A. M., Hori, O., and Samali, A. (2010). Cellular stress responses: cell survival and cell death. Int. J. Cell Biol. 2010:214074. doi: 10.1155/2010/214074

Galardo, M. N., Riera, M. F., Pellizzari, E. H., Cigorraga, S. B., and Meroni, S. B. (2007). The AMP-activatedprotein kinase activator, 5-aminoimidazole4-carboxamide-1-b-D-ribonucleoside, regulates lactate production in rat Sertolicells. J. Mol. Endocrinol. 39, 279-288. doi: 10.1677/JME-07-0054

Goransson, O., McBride, A., Hawley, S. A., Ross, F. A., Shpiro, N., Foretz, M., et al. (2007). Mechanism of action of A-769662, a valuable tool for 
activation of AMP-activated protein kinase. J. Biol. Chem. 282, 32549-32560. doi: 10.1074/jbc.m706536200

Greer, E. L., Dowlatshahi, D., Banko, M. R., Villen, J., Hoang, K., Blanchard, D., et al. (2007). An AMPK-FOXO pathway mediates longevity induced by a novel method of dietary restriction in C.elegans. Curr. Biol. 17, 1646-1656. doi: 10.1016/j.cub.2007.08.047

Guevelou, E., Huvet, A., Galindo-Sanchez, C. E., Milan, M., Quillien, V., Daniel, J. Y., et al. (2013). Sex-specific regulation of AMP-activated protein kinase (AMPK) in the Pacific oyster Crassostreagigas. Biol. Reprod. 89, 100. doi: 10.1095/biolreprod.113.109728

Hardie, D. G. (2006). Neither LKB1 nor AMPK are the direct targets of metformin. Gastroenterology 131, 973, author reply 974-975. doi: 10.1053/j.gastro.2006.07.032

Hardie, D. G. (2011). AMP-activated protein kinase: an energy sensor that regulates all aspects of cell function. Genes Dev. 25, 1895-1908. doi: $10.1101 /$ gad.17420111

Hardie, D. G., and Ashford, M. L. (2014). AMPK: regulating energy balance at the cellular and whole body levels. Physiology 29, 99-107. doi: $10.1152 /$ physiol.00050.2013

Hardie, D. G., Scott, J. W., Pan, D. A., and Hudson, E. R. (2003). Management of cellular energy by the AMP-activated protein kinase system. FEBS Lett. 546, 113-120. doi: 10.1016/S0014-5793(03)00560-X

Hawley, S. A., Boudeau, J., Reid, J. L., Mustard, K. J., Udd, L., Makela, T. P., et al. (2003). Complexes between the LKB1 tumor suppressor, STRAD $\alpha / \beta$ and MO25 $\alpha / \beta$ are upstream kinases in the AMP-activated protein kinase cascade. J. Biol. 2:28. doi: 10.1186/1475-4924-2-28

Hawley, S. A., Ross, F. A., Chevtzoff, C., Green, K. A., Evans, A., Fogarty, S., et al. (2010). Use of cells expressing gamma subunit variants to identify diverse mechanisms of AMPK activation. Cell Metab. 11, 554-565. doi: 10.1016/j.cmet.2010.04.001

Hudson, E. R., Pan, D. A., James, J., Lucocq, J. M., Hawley, S. A., Green, K. A., et al. (2003). A novel domain in AMP-activated protein kinase causes glycogen storage bodies similar to those seen in hereditary cardiac arrhythmias. Curr. Biol. 13, 861-866. doi: 10.1016/s0960-9822(03)00249-5

Hurtado de Llera, A., Martin-Hidalgo, D., Gil, M. C., Garcia-Marin, L. J., and Bragado, M. J. (2012). AMP-activated kinase AMPK is expressed in boar spermatozoa and regulates motility. PLoS ONE 7:e38840. doi: 10.1371/journal.pone.0038840

Hurtado de Llera, A., Martin-Hidalgo, D., Gil, M. C., Garcia-Marin, L. J., and Bragado, M. J. (2015). AMPK up-activation reduces motility and regulates other functions of boar spermatozoa. Mol. Hum. Reprod. 21, 31-45. doi: 10.1093/molehr/gau091

Hurtado de Llera, A., Martin-Hidalgo, D., Rodriguez-Gil, J. E., Gil, M. C., Garcia-Marin, L. J., and Bragado, M. J. (2013). AMP-activated kinase, AMPK, is involved in the maintenance of plasma membrane organization in boar spermatozoa. Biochim. Biophys. Acta 1828, 2143-2151. doi: 10.1016/j.bbamem.2013.05.026

Jeon, S. M., Chandel, N. S., and Hay, N. (2012). AMPK regulates NADPH homeostasis to promote tumour cell survival during energy stress. Nature 485, 661-665. doi: 10.1038/nature11066

Jiang, R., and Carlson, M. (1997). The Snf1 protein kinase and its activating subunit, Snf4, interact with distinct domains of the Sip1/Sip2/Gal83 component in the kinase complex. Mol. Cell Biol. 17, 2099-2106. doi: $10.1128 / \mathrm{mcb} .17 .4 .2099$

Kalinina, E. V., Chernov, N. N., and Saprin, A. N. (2008). Involvement of thio, peroxi-, and glutaredoxins in cellular redox-dependent processes. Biochem. Mosc. 73, 1493-1510. doi: 10.1134/S0006297908130099

Kim, J. E., Kim, Y. W., Lee, I. K., Kim, J. Y., Kang, Y. J., and Park, S. Y. (2008). AMP-activated protein kinase activation by 5 -aminoimidazole-4-carboxamide1-beta-D-ribofuranoside (AICAR) inhibits palmitate-induced endothelial cell apoptosis through reactive oxygen species suppression. J. Pharmacol. Sci. 106, 394-403. doi: 10.1254/jphs.FP0071857

King, T. D., Song, L., and Jope, R. S. (2006). AMP-activated protein kinase (AMPK) activating agents cause dephosphorylation of Akt and glycogen synthase kinase-3. Biochem. Pharmacol. 71, 1637-1647. doi: 10.1016/j.bcp.2006.03.005

Kong, Q., and Lin, C. L. (2010). Oxidative damage to RNA: mechanisms, consequences, and diseases. Cell. Mol. Life Sci. 67, 1817-1829. doi: 10.1007/s00018-010-0277-y
Lee, H., Cho, J. S., Lambacher, N., Lee, J., Lee, S. J., Lee, T. H., et al. (2008). The Caenorhabditiselegans AMP-activated protein kinase AAK-2 is phosphorylated by LKB1 and is required for resistance to oxidative stress and for normal motility and foraging behavior. J. Biol. Chem. 283, 14988-14993. doi: 10.1074/jbc.M709115200

Li, X. N., Song, J., Zhang, L., LeMaire, S. A., Hou, X., Zhang, C., et al. (2009). Activation of the AMPK-FOXO3 pathway reduces fatty acid-induced increase in intracellular reactive oxygen species by upregulating thioredoxin. Diabetes 58, 2246-2257. doi: 10.2337/db08-1512

Martin, L. J. (2010). Mitochondrial and cell death mechanisms in neurodegenerative diseases. Pharmaceuticals 3, 839-915. doi: $10.3390 / \mathrm{ph} 3040839$

Mojica-Villegas, M. A., Izquierdo-Vega, J. A., Chamorro-Cevallos, G., and Sanchez-Gutierrez, M. (2014). Protective effect of resveratrol on biomarkers of oxidative stress induced by iron/ascorbate in mouse spermatozoa. Nutrients 6 , 489-503. doi: 10.3390/nu6020489

Momcilovic, M., Hong, S. P., and Carlson, M. (2006). Mammalian TAK1 activates Snf1 protein kinase in yeast and phosphorylates AMP-activated protein kinase in vitro. J. Biol. Chem. 281, 25336-25343. doi: 10.1074/jbc.m604399200

Moreno, D., Knecht, E., Viollet, B., and Sanz, P. (2008). A-769662, a novel activator of AMP-activated protein kinase, inhibits non-proteolytic components of the $26 \mathrm{~S}$ proteasome by an AMPK-independent mechanism. FEBS Lett. 582, 2650-2654. doi: 10.1016/.j.febslet.2008.06.044

Myers, J. M., and Myers, C. R. (2009). The effects of hexavalent chromium on thioredoxin reductase and peroxiredoxins in human bronchial epithelial cells. Free Radic. Biol. Med. 47, 1477-1485. doi: 10.1016/j.freeradbiomed.2009.08.015

Nasrolahi, O., Khaneshi, F., Rahmani, F., and Razi, M. (2013). Honey and metformin ameliorated diabetes-induced damages in testis of rat; correlation with hormonal changes. Iran J. Reprod. Med. 11, 1013-1020.

Navarro, A., and Boveris, A. (2010). Brain mitochondrial dysfunction in aging, neurodegeneration, and Parkinson's disease. Front. Aging Neurosci. 2:34. doi: 10.3389/fnagi.2010.00034

Nguyen, T. M., Alves, S., Grasseau, I., Metayer-Coustard, S., Praud, C., Froment, P., et al. (2014). Central role of 5'-AMP-activated protein kinase in chicken sperm functions. Biol. Reprod. 91, 121. doi: 10.1095/biolreprod.114.121855

Nguyen, T. M., Seigneurin, F., Froment, P., Combarnous, Y., and Blesbois, E. (2015). The 5'-AMP-Activated Protein Kinase (AMPK) Is Involved in the augmentation of antioxidant defenses in cryopreserved chicken sperm. PLoS ONE 10:e0134420. doi: 10.1371/journal.pone.0134420

Pacher, P., Beckman, J. S., and Liaudet, L. (2007). Nitric oxide and peroxynitrite in health and disease. Physiol. Rev. 87, 315-424. doi: 10.1152/physrev.00029.2006

Polekhina, G., Gupta, A., Michell, B. J., van Denderen, B., Murthy, S., Feil, S. C., et al. (2003). AMPK beta subunit targets metabolic stress sensing to glycogen. Curr. Biol. 13, 867-871. doi: 10.1016/S0960-9822(03)00292-6

Rato, L., Alves, M. G., Socorro, S., Duarte, A. I., Cavaco, J. E., and Oliveira, P. F. (2012). Metabolic regulation is important for spermatogenesis. Nat. Rev. Urol. 9, 330-338. doi: 10.1038/nrurol.2012.77

Sanders, M. J., Ali, Z. S., Hegarty, B. D., Heath, R., Snowden, M. A., and Carling, D. (2007). Defining the mechanism of activation of AMP-activated protein kinase by the small molecule A-769662, a member of the thienopyridone family. J. Biol. Chem. 282, 32539-32548. doi: 10.1074/jbc.m706543200

Scott, J. W., Hawley, S. A., Green, K. A., Anis, M., Stewart, G., Scullion, G. A., et al. (2004). CBS domains form energy-sensing modules whose binding of adenosine ligands is disrupted by disease mutations. J. Clin. Invest. 113, 274-284. doi: 10.1172/JCI19874

Scott, J. W., Ling, N., Issa, S. M., Dite, T. A., O'Brien, M. T., Chen, Z. P., et al. (2014). Small molecule drug A-769662 and AMP synergistically activate naive AMPK independent of upstream kinase signaling. Chem. Biol. 21, 619-627. doi: 10.1016/j.chembiol.2014.03.006

Scott, J. W., van Denderen, B. J., Jorgensen, S. B., Honeyman, J. E., Steinberg, G. R., Oakhill, J. S., et al. (2008). Thienopyridone drugs are selective activators of AMP-activated protein kinase beta1-containing complexes. Chem. Biol. 15, 1220-1230. doi: 10.1016/j.chembiol.2008.10.005

Shabani Nashtaei, M., Amidi, F., Sedighi Gilani, M. A., Aleyasin, A., Bakhshalizadeh, S., Naji, M., et al. (2016). Protective features of resveratrol on human spermatozoa cryopreservation may be mediated through 5' AMPactivated protein kinase activation. Andrology 5, 313-326. doi: 10.1111/andr. 12306 
Shin, S. M., Cho, I. J., and Kim, S. G. (2009). Resveratrol protects mitochondria against oxidative stress through AMP-activated protein kinase-mediated glycogen synthase kinase-3 $\beta$ inhibition downstream of poly(ADP-ribose) polymerase-LKB1 pathway. Mol. Pharmacol. 76, 884-895. doi: 10.1124/mol.109.058479

Sundaresan, M., Yu, Z. X., Ferrans, V. J., Irani, K., and Finkel, T. (1995). Requirement for generation of $\mathrm{H}_{2} \mathrm{O}_{2}$ for platelet-derived growth factor signal transduction. Science 270, 296-299. doi: 10.1126/science.270.5234.296

Svechnikov, K., Spatafora, C., Svechnikova, I., Tringali, C., and Soder, O. (2009). Effects of resveratrol analogs on steroidogenesis and mitochondrial function in rat Leydig cells in vitro. J. Appl. Toxicol. 29, 673-680. doi: 10.1002/jat.1456

Tanwar, P. S., Kaneko-Tarui, T., Zhang, L., and Teixeira, J. M. (2012). Altered LKB1/AMPK/TSC1/TSC2/mTOR signaling causes disruption of Sertoli cell polarity and spermatogenesis. Hum. Mol. Genet. 21, 4394-4405. doi: $10.1093 / \mathrm{hmg} / \mathrm{dds} 272$

Tartarin, P., Guibert, E., Toure, A., Ouiste, C., Leclerc, J., Sanz, N., et al. (2012). Inactivation of AMPK $\alpha 1$ induces asthenozoospermia and alters spermatozoa morphology. Endocrinology 153, 3468-3481. doi: 10.1210/en.2011-1911

Thornton, C., Snowden, M. A., and Carling, D. (1998). Identification of a novel AMP-activated protein kinase beta subunit isoform that is highly expressed in skeletal muscle. J. Biol. Chem. 273, 12443-12450. doi: 10.1074/jbc.273.20. 12443

Towler, M. C., Fogarty, S., Hawley, S. A., Pan, D. A., Martin, D. M., Morrice, N. A., et al. (2008). A novel short splice variant of the tumour suppressor LKB1 is required for spermiogenesis. Biochem. J. 416, 1-14. doi: 10.1042/BJ200 81447

Um, J. H., Park, S. J., Kang, H., Yang, S., Foretz, M., McBurney, M. W., et al. (2010). AMP-activated protein kinase-deficient mice are resistant to the metabolic effects of resveratrol. Diabetes 59, 554-563. doi: $10.2337 / \mathrm{db}$ 09-0482

Viollet, B., Guigas, B., Sanz Garcia, N., Leclerc, J., Foretz, M., and Andreelli, F. (2012). Cellular and molecular mechanisms of metformin: an overview. Clin. Sci. 122, 253-270. doi: 10.1042/CS20110386

Wang, X. R., Zhang, M. W., Chen, D. D., Zhang, Y., and Chen, A. F. (2011). AMP-activated protein kinase rescues the angiogenic functions of endothelial progenitor cells via manganese superoxide dismutase induction in type 1 diabetes. Am. J. Physiol. Endocrinol. Metab. 300, E1135-E1145. doi: 10.1152/ajpendo.00001.2011

Williams, A. C., and Ford, W. C. (2004). Functional significance of the pentose phosphate pathway and glutathione reductase in the antioxidant defenses of human sperm. Biol. Reprod. 71, 1309-1316. doi: 10.1095/biolreprod.104.028407

Woods, A., Dickerson, K., Heath, R., Hong, S. P., Momcilovic, M., Johnstone, S. R., et al. (2005). $\mathrm{Ca}^{2+} /$ calmodulin-dependent protein kinase kinase- $\beta$ acts upstream of AMP-activated protein kinase in mammalian cells. Cell Metab. 2, 21-33. doi: 10.1016/j.cmet.2005.06.005

Woods, A., Johnstone, S. R., Dickerson, K., Leiper, F. C., Fryer, L. G., Neumann, D., et al. (2003). LKB1 is the upstream kinase in the AMP-activated protein kinase cascade. Curr. Biol. 13, 2004-2008. doi: 10.1016/j.cub.2003.10.031

Wu, S. B., and Wei, Y. H. (2012). AMPK-mediated increase of glycolysis as an adaptive response to oxidative stress in human cells: implication of the cell survival in mitochondrial diseases. Biochim. Biophys. Acta 1822, 233-247. doi: 10.1016/j.bbadis.2011.09.014

Zang, M., Xu, S., Maitland-Toolan, K. A., Zuccollo, A., Hou, X., Jiang, B., et al. (2006). Polyphenols stimulate AMP-activated protein kinase, lower lipids, and inhibit accelerated atherosclerosis in diabetic LDL receptor-deficient mice. Diabetes 55, 2180-2191. doi: 10.2337/db05-1188

Zhou, G., Myers, R., Li, Y., Chen, Y., Shen, X., Fenyk-Melody, J., et al. (2001). Role of AMP-activated protein kinase in mechanism of metformin action. J. Clin. Invest. 108, 1167-1174. doi: 10.1172/JCI13505

Conflict of Interest Statement: The author declares that the research was conducted in the absence of any commercial or financial relationships that could be construed as a potential conflict of interest.

Copyright (C) 2017 Nguyen. This is an open-access article distributed under the terms of the Creative Commons Attribution License (CC BY). The use, distribution or reproduction in other forums is permitted, provided the original author $(s)$ or licensor are credited and that the original publication in this journal is cited, in accordance with accepted academic practice. No use, distribution or reproduction is permitted which does not comply with these terms. 